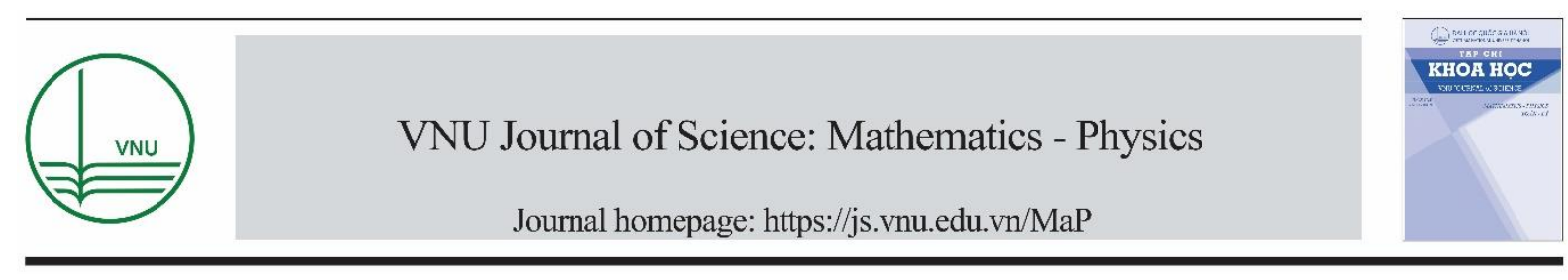

\title{
Stability of Arbitrarily Switched Discrete-time Linear Singular Systems of Index-1
}

\author{
Pham Thi Linh* \\ VNU University of Science, 334 Nguyen Trai, Thanh Xuan, Hanoi, Vietnam
}

Received 22 December 2018

Revised 27 December 2018; Accepted 28 December 2018

\begin{abstract}
In this paper, the index-1 notion for arbitrarily switched discrete-time linear singular systems (SDLS) has been introduced. Based on the Bohl exponents of SDLS as well as properties of associated positive switched systems, some necessary and sufficient conditions have been established for exponential stability.

Keywords: Switched system, linear discrete-time singular system, positive system, index-1 system.
\end{abstract}

\section{Introduction}

Recently there has been a great interest in arbitrarily switched discrete-time linear singular systems due to their importance in both theoretical and practical aspects, see [1-4], and the references therein. Consider a switched system consisting of a set of subsystems and a rule that describes switching among them. It is well known that, even if all linear descriptor subsystems are stable but inappropriate switching may make the whole system unstable. On the other hand, since abrupt changes in system dynamics may be caused by unpredictable environmental factors or component failures, it is important to require the stability for some real-life switched systems under arbitrary switching. It should be noted that although there are a few works devoted to stability analysis of SDLS, see [1, 3-5], to our best of knowledge, the problem of investigating the stability for such switched systems via their Bohl exponents or properties of associated positive switched systems has not yet been studied before. Thus, this work was intended as an attempt to fill this gap.

\section{Switched discrete-time linear singular systems of index-1}

Consider the following autonomous SDLS of the form:

\footnotetext{
${ }^{*}$ Tel.: 84-336112702.

Email: linhpt1803@gmail.com

https//doi.org/ 10.25073/2588-1124/vnumap.4312
} 


$$
E_{\sigma(k+1)} x(k+1)=A_{\sigma(k)} x(k)
$$

where $\sigma: \mathrm{N} \cup\{0\} \rightarrow I_{N}:=\{1,2, . ., N\}, N \in \mathrm{N}$, is a switching signal taking values in the finite set $I_{N}$; $E_{i}, A_{i} \in \mathrm{R}^{n \times n}$ are given matrices, and $x(k) \in \mathrm{R}^{n}$ are unknown vector for al $k \in \mathrm{N}$. Suppose that the matrices $E_{i}$ are singular for all $i=1,2, . ., N$.

We remark that in some works on SDLS [4, 6], instead of (1), a simpler system of the form

$E_{\sigma(k)} x(k+1)=A_{\sigma(k)} x(k)$,

can be considered. Moreover, all the techniques developed in this paper can easily be applied to the above mentioned SDLS.

Definition 1 System (1) is called an arbitrarily switched singular system of index-1 (shortly, index1 SDLS) if it satisfies the following conditions

(i) $\operatorname{rank} E_{i}=r<n$;

(ii) $S_{i j} \cap \operatorname{ker} E_{i}=\{0\} \forall i, j$, where $S_{i j}=A_{i}^{-1}\left(\operatorname{Im} E_{j}\right)=\left\{\xi: A_{i} \xi \in \operatorname{Im} E_{j}\right\}$.

From condition (ii) in Definition 1 we show that

$$
S_{i j} \oplus \operatorname{ker} E_{i}=\mathrm{R}^{n} \forall i, j \in\{1,2, . ., N\} .
$$

Indeed, put $W_{i j}=\operatorname{Im} A_{i} \cap \operatorname{Im} E_{j}$. Then consider linear operators $T_{i j}: S_{i j} \rightarrow W_{i j}$, defined by $T_{i j} x=A_{i j} x$, we can easily show that $\operatorname{ker} T_{i j}=\operatorname{ker} A_{i}$. According to [7] we have

$\operatorname{dim} S_{i j}=\operatorname{dim} W_{i j}+\operatorname{dim} \operatorname{ker} T_{i j}=\operatorname{dim} W_{i j}+\operatorname{dim} \operatorname{ker} A_{i}$.

On the other hand

$$
\begin{aligned}
\operatorname{dim} W_{i j} & =\operatorname{dim}\left(\operatorname{Im} A_{i} \cap \operatorname{Im} E_{j}\right) \\
& =\operatorname{dim} \operatorname{Im} A_{i}+\operatorname{dim} \operatorname{Im} E_{j}-\operatorname{dim}\left(\operatorname{Im} A_{i} \cup \operatorname{Im} E_{j}\right) .
\end{aligned}
$$

From last the relation we get

$$
\begin{aligned}
\operatorname{dim} S_{i j} & =\operatorname{dim} \operatorname{Im} A_{i}+\operatorname{dim} \operatorname{Im} E_{j}-\operatorname{dim}\left(\operatorname{Im} A_{i} \cup \operatorname{Im} E_{j}\right)+\operatorname{dim}\left(\operatorname{ker} A_{i}\right) \\
& =n+r-\operatorname{dim}\left(\operatorname{Im} A_{i} \cup \operatorname{Im} E_{j}\right) .
\end{aligned}
$$

This relation shows that $\operatorname{dim} S_{i j} \geq r$. Moreover, from condition (ii) in Definition 1 we have $\operatorname{dim} S_{i j} \leq r$. Hence $\operatorname{dim} S_{i j}=r$, i.e., $S_{i j} \oplus \operatorname{ker} E_{i}=\mathrm{R}^{n}$.

Define the matrix $V_{i j}=\left\{s_{i j}^{1}, \ldots, s_{i j}^{r}, h_{i}^{r+1}, \ldots, h_{i}^{n}\right\}$, whose columns form bases of $S_{i j}$ and $\operatorname{ker} E_{i}$, respectively, and $Q=\operatorname{diag}\left(O_{r}, I_{n-r}\right), P=I_{n}-Q$. Here $O_{r}$ is the $r \times r$ zero matrix and $I_{m}$ stands for the $m \times m$ identity matrix.

Then the matrix $Q_{i j}=V_{i j} Q V_{i j}^{-1}$ defines a projection onto ker $E_{i}$ along $S_{i j}$ and $P_{i j}=I_{n}-Q_{i j}$ is the projection onto $S_{i j}$ along ker $E_{i}$.

Using similar arguments as in [8-11] we can prove the following results.

Theorem 1 For index-1 SDLS (1), the following assertions hold.

(i) $G_{i j k}=E_{j}+A_{i} V_{i j} Q V_{j k}^{-1}$ is non-singular for all $i, j, k \in\{1,2, . ., N\}$; 
(ii) $E_{j} P_{j k}=E_{j}$;

(iii) $P_{j k}=G_{i j k}^{-1} E_{j}$;

(iv) $V_{j k}^{-1} G_{i j k}^{-1} A_{i} V_{i j} Q=Q$.

\section{Proof.}

(i) Assume that $x \in \operatorname{ker} G_{i j k}$, we have $0=G_{i j k} x=\left(E_{j}+A_{i} V_{i j} Q V_{j k}^{-1}\right) x=E_{j} x+A_{i} V_{i j} Q V_{j k}^{-1} x$.Then $E_{j} x=-A_{i} V_{i j} Q V_{j k}^{-1} x$, thus $V_{i j} Q V_{j k}^{-1} x \in S_{i, j}$. Furthermore, $V_{i j} Q V_{j k}^{-1} x=V_{i j} Q V_{i j}^{-1} V_{i j} V_{j k}^{-1} x=Q_{i j} V_{i j} V_{j k}^{-1} x \in \operatorname{ker} E_{i}$. Since $S_{i j} \cap \operatorname{ker} E_{i}=\{0\}$ we get $V_{i j} Q V_{j k}^{-1} x=0$, thus $E_{j} x=-A_{i} V_{i j} Q V_{j k}^{-1} x=0$, hence $x \in \operatorname{ker} E_{j}=\operatorname{Im} Q_{j k}$, i.e., $x=Q_{j k} x$. On the other hand, from the relation $Q_{j k} x=V_{j k} V_{i j}^{-1} V_{i j} Q V_{j k}^{-1} x=0$, we have $x=Q_{j k} x=0$. It means that $\operatorname{ker} G_{i j k}=\{0\}$, i.e., the matrix $G_{i j k}$ is non-singular.

(ii) Since $Q_{j k}$ is the projection onto $\operatorname{ker} E_{j}$ then we have $E_{j} Q_{j k}=0$, i.e., $E_{j}=E_{j}\left(P_{j k}+Q_{j k}\right)=E_{j} P_{j k}$.

(iii) From relation $G_{i j k} P_{j k}=\left(E_{j}+A_{i} V_{i j} Q V_{j k}^{-1}\right) V_{j k} P V_{j k}^{-1}=E_{j} P_{j k}+A_{i} V_{i j} Q P V_{j k}^{-1}=E_{j}$, we get $P_{j k}=G_{i j k}^{-1} E_{j}$.

(iv) From formula of $G_{i j k}=E_{j}+A_{i} V_{i j} Q V_{j k}^{-1}$ we have $G_{i j k} V_{j k}=E_{j} V_{j k}+A_{i} V_{i j} Q$, thus $A_{i} V_{i j} Q=G_{i j k} V_{j k}-E_{j} V_{j k}$.

The last assertion follows from relations:

$$
\begin{aligned}
V_{j k}^{-1} G_{i j k}^{-1} A_{i} V_{i j} Q & =V_{j k}^{-1} G_{i j k}^{-1}\left(G_{i j k} V_{j k}-E_{j} V_{j k}\right) \\
& =V_{j k}^{-1} V_{j k}-V_{j k}^{-1} G_{i j k}^{-1} E_{j} V_{j k} \\
& =I_{n}-V_{j k}^{-1} P_{j k} V_{j k} \\
& =Q .
\end{aligned}
$$

Theorem 1 is proved.

Using items (iii), and (iv) of Theorem 1, we get

$$
\begin{aligned}
& \bar{A}_{i j k}:=V_{j k}^{-1} G_{i j k}^{-1} A_{i} V_{i j}=\left(\begin{array}{cc}
\bar{A}_{i j k}^{1} & O \\
O & I_{n-r}
\end{array}\right) ; \\
& \bar{E}_{i j k}:=V_{j k}^{-1} G_{i j k}^{-1} E_{j} V_{j k}=\left(\begin{array}{cc}
I_{r} & O \\
O & O_{n-r}
\end{array}\right) .
\end{aligned}
$$

Theorem 2 The index-1 SDLS (1) has a unique solution with $x(0)=x_{0} \in \mathrm{R}$ if and only if $x_{0} \in S_{\sigma(0) \sigma(1)}$ , i.e., the initial condition $x_{0}$ is consistent. In this case, the following solution formula holds.

$x(k)=V_{\sigma(k) \sigma(k+1)} \bar{A}_{\sigma(k-1) \sigma(k) \sigma(k+1)} \ldots \bar{A}_{\sigma(0) \sigma(1) \sigma(2)} V_{\sigma(0) \sigma(1)}^{-1} x(0)$.

\section{Proof.}

Multiplying both sides of system (1) by $V_{\sigma(k+1) \sigma(k+2)}^{-1} G_{\sigma(k) \sigma(k+1) \sigma(k+2)}^{-1}$, and using the transformation $\bar{x}(k)=V_{\sigma(k) \sigma(k+1)}^{-1} x(k)$, we get 


$$
\bar{E}_{\sigma(k) \sigma(k+1) \sigma(k+2)} \bar{x}(k+1)=\bar{A}_{\sigma(k) \sigma(k+1) \sigma(k+2)} \bar{x}(k) .
$$

Putting $\bar{x}(k):=\left(v(k)^{T}, w(k)^{T}\right)^{T}$, where $v(k) \in \mathrm{R}^{r}, w(k) \in \mathrm{R}^{n-r}$, we can reduce system (3) to the following systems

$$
\left\{\begin{array}{l}
v(k+1)=\bar{A}_{\sigma(k) \sigma(k+1) \sigma(k+2)}^{1} v(k), \\
w(k)=0
\end{array}\right.
$$

System (4) has the solution

$$
\begin{aligned}
& v(k)=\bar{A}_{\sigma(k-1) \sigma(k) \sigma(k+1)}^{1} \ldots \bar{A}_{\sigma(0) \sigma(1) \sigma(2)}^{1} v(0), \\
& w(k)=0,
\end{aligned}
$$

hence the solution of system (1) can be written as

$$
\begin{aligned}
x(k) & =V_{\sigma(k) \sigma(k+1)} \bar{x}(k) \\
& =V_{\sigma(k) \sigma(k+1)}\left(\begin{array}{c}
v(k) \\
w(k)
\end{array}\right) \\
& =V_{\sigma(k) \sigma(k+1)} \bar{A}_{\sigma(k-1) \sigma(k) \sigma(k+1)} \ldots \bar{A}_{\sigma(0) \sigma(1) \sigma(2)}\left(\begin{array}{c}
v(0) \\
0
\end{array}\right) \\
& =V_{\sigma(k) \sigma(k+1)} \bar{A}_{\sigma(k-1) \sigma(k) \sigma(k+1)} \ldots \bar{A}_{\sigma(0) \sigma(1) \sigma(2)} V_{\sigma(0) \sigma(1)}^{-1} x(0) .
\end{aligned}
$$

\section{Stability of linear switched singular systems of index-1}

Suppose that system (1) is of index-1 and the initial condition $x_{0}$ is consistent.

Definition 2 System (1) is called exponentially stable if there exist a positive constant $\gamma$ and a constant $0 \leq \lambda<1$ such that such that for all switching signals and all solutions $x$ of (1) the following inequality holds

$$
\|x(k)\| \leq \gamma \lambda^{k}\left\|x_{0}\right\| \forall k \geq 0 .
$$

\subsection{Bohl exponents and exponential stability}

To define Bohl exponent for system (1), we first construct the so-called one-step solution operator $\Phi_{\sigma}(k, k-1)$ from $x(k-1)$ to $x(k)$.

$$
\begin{aligned}
x(k) & =V_{\sigma(k) \sigma(k+1)} \bar{x}(k) \\
& =V_{\sigma(k) \sigma(k+1)}\left(\begin{array}{c}
v(k) \\
w(k)
\end{array}\right) \\
& =V_{\sigma(k) \sigma(k+1)} \bar{A}_{\sigma(k-1) \sigma(k) \sigma(k+1)} \bar{x}(k-1) \\
& =V_{\sigma(k) \sigma(k+1)} \bar{A}_{\sigma(k-1) \sigma(k) \sigma(k+1)} V_{\sigma(k-1) \sigma(k)}^{-1} x(k-1) .
\end{aligned}
$$

Then put $\Phi_{\sigma}(k, k-1):=\Phi_{\sigma(k-1) \sigma(k) \sigma(k+1)}=V_{\sigma(k) \sigma(k+1)} \bar{A}_{\sigma(k-1) \sigma(k) \sigma(k+1)} V_{\sigma(k-1) \sigma(k)}^{-1}$ we get the following one-step solution operator 
$x(k)=\Phi_{\sigma}(k, k-1) x(k-1)$.

Hence we can define the state transition matrix as

$\Phi_{\sigma}(i, j):=\Phi_{\sigma(i-1) \sigma(i) \sigma(i+1)} \ldots \Phi_{\sigma(j) \sigma(j+1) \sigma(j+2)}, \forall i \geq j \geq 0$.

Definition 3 Assume that system (1) is of index-1 and $\Phi_{\sigma}(i, j)$ is the state transition matrix. Then Bohl exponent for system (1) is defined as follows:

$\kappa_{B}=\inf \left\{w \in \mathrm{R}: \exists M_{w}:\left\|\Phi_{\sigma}(i, j)\right\| \leq M_{w} \cdot w^{i-j}, \forall \sigma, i \geq j \geq 0\right\}$.

To show the existence of Bohl exponent $\kappa_{B}$ for system (1) we will prove that the set

$$
S=\left\{w \in \mathrm{R}: \exists M_{w}:\left\|\Phi_{\sigma}(i, j)\right\| \leq M_{w} \cdot w^{i-j}, \forall \sigma, i \geq j \geq 0\right\},
$$

is non-empty and bounded from below.

Indeed, from the formula $\Phi_{i j k}=V_{j k} \bar{A}_{i j k} V_{i j}^{-1}, i, j, k \in\{1,2, . ., N\}$, we see that the set of matrices $\Phi_{i j k}$ is finite, then there exists a positive constant $\gamma>0$ such that

$$
\gamma=\max _{i, j, k \in\{1,2, . ., N\}}\left\|\Phi_{i j k}\right\| .
$$

Thus we obtain that

| $\Phi_{\sigma}(i, j) \| \leq \gamma^{i-j}, \forall \sigma, i \geq j \geq 0$,

hence $\gamma \in S$. Besides, for all $w \in S$ we have $w \geq 0$. It follows that the set $S$ is non-empty and bounded from below.

Lemma 1 Assume that system (1) is of index-1 and $\Phi_{\sigma}(i, j)$ is the state transition matrix. Then

$$
\kappa_{B}=\lim _{i \rightarrow \infty} \max _{\sigma}\left\|\Phi_{\sigma}(i, 0)\right\|^{\frac{1}{i}} .
$$

\section{Proof.}

We carry the proof of Lemma 1 in 3 steps.

Step 1.We show the existence of the limit in (5)

Put $a_{i}=\max _{\sigma} \Phi_{\sigma}(i, 0) \|$. Then we have $a_{i+j} \leq a_{i} a_{j}$ for all $i, j \geq 0$. According to Polya-Szego [12] we obtain that $\lim _{i \rightarrow \infty} a_{i}^{\frac{1}{i}}$ exists. It means that the limit in (5) exists.

Step 2.Put $\kappa_{1}=\lim _{i \rightarrow \infty} \max _{\sigma} \Phi_{\sigma}(i, 0) \|^{\frac{1}{i}}$. We prove $\kappa_{1} \leq \kappa_{B}$.

Since $\kappa_{B}=\inf S$ then for all $\epsilon>0$ there exists $w_{\epsilon} \in S$ such that $w_{\epsilon}<\kappa_{B}+\epsilon$, i.e., there exists $M_{w_{\epsilon}}$ such that

| $\Phi_{\sigma}(i, 0) \mid<M_{w_{\epsilon}}\left(\kappa_{B}+\epsilon\right)^{i-j}, \forall \sigma, i \geq 0$.

It follows

$\lim _{i \rightarrow \infty} \max _{\sigma}\left\|\Phi_{\sigma}(i, 0)\right\|^{\frac{1}{i}} \leq \kappa_{B}+\epsilon$.

Then we have 
Step 3. We prove $\kappa_{B} \leq \kappa_{1}$.

From the definition of $\kappa_{1}$, for all $\epsilon>0$ there exists $T>0$ such that

$\left|a_{i}^{\frac{1}{i}}-\kappa_{1}\right|<\epsilon, \forall i>T$,

i.e.,

$$
\text { | } \Phi_{\sigma}(i, 0) \|<\left(\kappa_{1}+\epsilon\right)^{i}, \forall i>T, \forall \sigma .(6)
$$

We will show that there exists $\mathrm{M}>0$ such that

$$
\text { | } \Phi_{\sigma}(i, j) \|<M\left(\kappa_{1}+\epsilon\right)^{i-j}, \forall i>T, \forall \sigma .
$$

Indeed, when $i-j>T$, for every $\sigma$ we always have switching signal $\sigma^{*}$ such that $\Phi_{\sigma}(i, j)=\Phi_{\sigma^{*}}(i-j, 0)$. Hence we have

$\mid \Phi_{\sigma}(i, j)\|=\| \Phi_{\sigma^{*}}(i-j, 0) \|<\left(\kappa_{1}+\epsilon\right)^{i-j}, \forall i-j>T, \forall \sigma$.

When $i-j \leq T$, we have the following estimate

| $\Phi_{\sigma}(i, j) \|<\gamma^{i-j}=\left(\frac{\gamma}{\kappa_{1}+\epsilon}\right)^{i-j}\left(\kappa_{1}+\epsilon\right)^{i-j}$.

Choosing $M=\max \left\{1,\left(\frac{\gamma}{\kappa_{1}+\epsilon}\right)^{T}\right\}$, we get the inequality (7). It means that $\kappa_{B} \leq \kappa_{1}$.

Thus we obtain $\kappa_{B}=\kappa_{1}$.

Lemma 1 is proved.

Theorem 2 An index-1 SDLS (1) is exponentially stable if and only if $\kappa_{B}<1$.

Proof.

Necessity.Assume that system (1) is exponentially stable. It follows that there exist a positive constant $\mathrm{M}>0$ and $0<\omega<1$ such that

$$
\Phi_{\sigma}(i, j) \leq M \omega^{i-j} \forall \sigma, i \geq j \geq 0 \text {. }
$$

Thus, $\kappa_{B}<1$.

Sufficiency. Assume that $\kappa_{B}<1$. Then there exist $\epsilon>0$ and $\mathrm{M}>0$ such that $\omega=\kappa_{B}+\epsilon<1$ and $\Phi_{\sigma}(i, j) \leq M \omega^{i-j} \forall \sigma, i \geq j \geq 0$. It shows that system (1) is exponentially stable.

Theorem 2 is proved.

\subsection{Stability of positive linear switched singular systems of index-1}

In this Subsection, we investigate the stability of index-1 SDLS satisfying some positivity condition. Let $\mathcal{K}:=\left\{x=\left(x_{1}, x_{2}, \ldots, x_{r}\right)^{T}, x_{i} \geq 0\right\}$ be a positive octant in $\mathrm{R}^{r}, \operatorname{Int}(\mathcal{K})$ be the interior of $\mathcal{K}$. Consider an order unit norm $\|\cdot\|_{u}$, defined in [13], [14], and the corresponding order unit space $\left(\mathrm{R}^{r}, \mathcal{K}\|\|_{u}\right)$. 
Theorem 3 Assume that the matrices $\bar{A}_{i j k}^{1}$, determined by (2), are positive definite, and there exists a vector $\hat{v} \in \operatorname{Int}(\mathcal{K})$ such that $\hat{v}-\bar{A}_{i j k}^{1} \hat{v} \in \operatorname{Int}(P \mathcal{K})$ for all $i, j, k$. Then system (4) is exponentially stable, hence system (1) is also exponentially stable.

\section{Proof.}

Since $\hat{v}-\bar{A}_{i j k}^{1} \hat{v} \in \operatorname{Int}(P \mathcal{K})$ then there exists a $\delta_{i j k} \in\left(0\|\|_{\|} \|_{u}\right)$ such that the closed ball $B\left[\hat{v}-\bar{A}_{i j k}^{1} \hat{v}, \delta_{i j k}\right] \subseteq \mathcal{K} . \quad$ Since $\quad \hat{v}-\bar{A}_{i j k}^{1} \hat{v}-\frac{\delta_{i j k}}{\|\|_{\|}} \hat{v} \in B\left[\hat{v}-\bar{A}_{i j k}^{1} \hat{v}, \delta_{i j k}\right]$ we $\quad$ get $\quad \hat{v}-\bar{A}_{i j k}^{1} \hat{v}-\frac{\delta_{i j k}}{\| v_{u}} \hat{v} \geq 0 . \quad$ Let $\epsilon_{i j k}=\frac{\delta_{i j k}}{\| \hat{\nu}_{u}} \in(0,1)$, then $\bar{A}_{i j k}^{1} \hat{v} \leq\left(1-\epsilon_{i j}\right) \hat{v}$.

Put $\epsilon=\inf \left\{\epsilon_{i j k}, i, j, k \in\{1,2, \ldots, N\}\right\}$, we obtain $\bar{A}_{i j k}^{1} \hat{v} \leq(1-\epsilon) \hat{v}$ for all $i, j, k$. Using the positive definiteness of matrices $\bar{A}_{i j k}^{1}$ and the monotonicity of $\mathrm{I}$ ैㅐ ${ }_{u}$ we get

$$
\begin{aligned}
& \left\|\bar{A}_{\sigma(k-1) \sigma(k) \sigma(k+1)}^{1} \ldots \bar{A}_{\sigma(0) \sigma(1) \sigma(2)}^{1}\right\|_{\mathcal{L}\left(\mathrm{R}^{r}\|\|_{\hat{v}}\right)} \\
= & \left\|\bar{A}_{\sigma(k-1) \sigma(k) \sigma(k+1)}^{1} \ldots \bar{A}_{\sigma(0) \sigma(1) \sigma(2)}^{1} \hat{\imath}\right\|_{\hat{v}} \\
\leq & \left\|\bar{A}_{\sigma(k-1) \sigma(k) \sigma(k+1)}^{1} \ldots \bar{A}_{\sigma(1) \sigma(2) \sigma(3)}^{1}(1-\epsilon) \hat{\imath}\right\|_{\hat{v}} \\
= & (1-\epsilon)\left\|\bar{A}_{\sigma(k-1) \sigma(k) \sigma(k+1)}^{1} \ldots \bar{A}_{\sigma(1) \sigma(2) \sigma(3)}^{1} \hat{v}\right\|_{\hat{v}} \\
& \ldots \\
\leq & (1-\epsilon)^{k}\|\|_{\hat{v}}=(1-\epsilon)^{k} .
\end{aligned}
$$

According to [15], system (4) is exponentially stable. It follows that there exist finite positive constants $0<\lambda<1$ and $\gamma>0$ such that

$$
\mid v(k)\left\|\leq \gamma \lambda^{k}\right\| v(0) \| .
$$

Furthermore since the corresponding solution of system (1) is $x(k)=V_{\sigma(k) \sigma(k+1)}\left(v(k)^{T}, 0\right)^{T}$, we have

$$
\begin{aligned}
\|x(k)\| & \leq\left\|V_{\sigma(k) \sigma(k+1)}\right\|\left\|\left(v(k)^{T}, 0\right)^{T}\right\| \\
& \leq \gamma \lambda^{k}\left\|V_{\sigma(k-1)} D_{\sigma(k) \sigma(k-1)}\right\|\left\|\left(V_{\sigma(k) \sigma(k+1)} v(0)^{T}, 0\right)^{T}\right\| \\
& \leq \gamma \lambda^{k}\left\|V_{\sigma(k) \sigma(k+1)}\right\|\left\|V_{\sigma(0) \sigma(1)}^{-1} x(0)\right\| \\
& \leq \gamma \lambda^{k}\left\|V_{\sigma(k) \sigma(k+1)}\right\|\left\|V_{\sigma(0) \sigma(1)}^{-1}\right\|\|x(0)\| .
\end{aligned}
$$

Putting $\mu=\gamma \max _{i, j=1,2, ., N}\left\|V_{i j}\right\|\left\|V_{i j}^{-1}\right\|$, we have

The last relation shows that the solution of system (1) is exponentially stable.

Theorem 3 is proved.

Example 1 Put

$$
E_{1}=\left(\begin{array}{ccc}
2 & -3 & 0 \\
0 & 2 & 0 \\
0 & 0 & 0
\end{array}\right), \quad E_{2}=\left(\begin{array}{ccc}
3 & 2 & 0 \\
1 & 6 & 0 \\
0 & 0 & 0
\end{array}\right),
$$




$$
\begin{array}{ll}
A_{1}=\left(\begin{array}{ccc}
1 & -1 & 0 \\
0 & 1 & 0 \\
0 & 0 & 1
\end{array}\right), \quad A_{2}=\left(\begin{array}{lll}
2 & 1 & 0 \\
1 & 2 & 1 \\
0 & 0 & 1
\end{array}\right), \\
V_{11}=V_{12}=\left(\begin{array}{ccc}
1 & 0 & 0 \\
0 & 1 / 3 & 0 \\
0 & 0 & 1
\end{array}\right), \quad V_{21}=V_{22}=\left(\begin{array}{lll}
2 & 0 & 0 \\
0 & 2 & 0 \\
0 & 0 & 1
\end{array}\right) .
\end{array}
$$

We calculate the matrices $\bar{A}_{i j k}, i, j, k \in\{1,2\}$ as

$$
\begin{aligned}
& \bar{A}_{111}=\bar{A}_{112}=\left(\begin{array}{ccc}
1 / 2 & 1 / 12 & 0 \\
0 & 1 / 2 & 0 \\
0 & 0 & 1
\end{array}\right), \quad \bar{A}_{121}=\bar{A}_{122}=\left(\begin{array}{ccc}
3 / 16 & -1 / 12 & 0 \\
-1 / 4 & 1 / 6 & 0 \\
0 & 0 & 1
\end{array}\right), \\
& \bar{A}_{211}=\bar{A}_{212}=\left(\begin{array}{ccc}
7 / 8 & 1 / 3 & 0 \\
1 / 4 & 1 / 6 & 0 \\
0 & 0 & 1
\end{array}\right), \quad \bar{A}_{221}=\bar{A}_{222}=\left(\begin{array}{ccc}
5 / 8 & 1 / 8 & 0 \\
1 / 16 & 5 / 16 & 0 \\
0 & 0 & 1
\end{array}\right) .
\end{aligned}
$$

Clearly all the matrices $\bar{A}_{i j k}$ are positive definite. We choose $\hat{v}=(9,3)^{T} \in \operatorname{Int}(\mathcal{K})$ and find that $\hat{v}-\bar{A}_{i j k}^{1} \hat{v}$ are also inside $\operatorname{Int}(\mathcal{K})$. It means that this system satisfies all the condition of Theorem 3, thus it is exponentially stable.

\section{Acknowledgments}

The author is grateful to Professors Pham Ky Anh, Do Duc Thuan and Stefan Trenn for useful discussions leading to the formulation of the problem as well as the results obtained in this paper.

\section{References}

[1] D. Liberzon, S. Trenn, On stability of linear switched differential algebraic equations, Proc. IEEE 48th Conf. Decision Control, December (2009) 2156.

[2] Z. Sun, S.S. Ge, Stability Theory of Switched Dynamical Systems, Springer, London, 2011.

[3] S. Trenn and F. Wirth, Linear switched DAEs: Lyapunov exponents, a converse Lyapunov theorem, and Barabanov norms, In Decision and Control (CDC), IEEE 51 $1^{\text {st }}$ Annual Conference, (2012) 2666.

[4] G. Zhai, X. Xu, A unified approach to stability analysis of switched linear descriptor systems under arbitrary switching, Internat. J. Appl. Math. Comput. Sci., 20(2) (2010) 249.

[5] M. Darouach and M. Chadli, Admissibility and control of switched discrete-time singular systems, Syst. Sci. Control Engrg.: An Open Access J., 1:1 (2013) 43.

[6] G. Zhai, X. Xu, D. W.C. Ho, Stability of switched linear discrete-time descriptor systems: a new commutation condition, Internat. J. Contr., 85 (2012) 1779.

[7] S.H. Friedberg, A.J. Insel and L.E. Spence, Linear Algebra, Prentice Hall, New Jersey, 1989.

[8] P.K. Anh, P.T. Linh, Stability of periodically switched discrete-time linear singular systems, J. Difference Equ. Appl., 23(10) (2017) 1680.

[9] P.K. Anh, H.T.N. Yen, Floquet theorem for linear implicit nonautonomous difference systems, J. Math. Anal. Appl., 321 (2006) 921.

[10] P.K. Anh, N.H. Du, L.C. Loi, Singular difference equations: an overview, Vietnam J.Math., 35 (2007) 339.

[11] L.C. Loi, N.H. Du, and P.K. Anh, On linear implicit non-autonomous systems of difference equations, J. Difference Equ. Appl. 8 (2002) 1085.

[12] G. Polya and G. Szego, Problems and Theorems in Analysis I, Springer, Berlin, Heidelberg, 1998.

[13] B.Z. Vulikh, Introduction to the Theory of Cones in Normed Spaces, Izdat. Kalinin Univ., Kaliningrad, 1977.

[14] T.S. Doan, A. Kalauch, M. Klose, S. Siegmund, Stability of positive linear switched systems on ordered Banach spaces, Syst.and Contr. Lett.75 (2015) 14.

[15] H. Lin, P.J. Antsaklis, Stability and stabilizability of switched Linear Systems: a survey of recent results, IEEE Trans. Aut. Contr. 54 (2009) 308. 